\title{
Probabilistic teleportation of two-particle entangled state
}

\author{
Bao-Sen Shi*, Yun-Kun Jiang and Guang-Can Guo ${ }^{\dagger}$ \\ Lab. of Quantum Communication and Quantum Computation \\ Department of Physics \\ University of Science and Technology of China \\ Hefei, 230026, P.R.China
}

\begin{abstract}
In this report, two different probabilistic teleportations of a two-particle entangled state by pure entangled three-particle state are shown. Their successful probabilities are different.

03.65.Bz, 03.67.-a
\end{abstract}

*E-mail: drshi@ustc.edu.cn

†E-Mail: gcguo@ustc.edu.cn 
Quantum teleportation, proposed by Bennett et.al [1], is the process that transmits an unknown two-state particle, or a qubit from a sender (Alice) to a receiver (Bob) via a quantum channel with the help of some classical information. In their scheme, such a quantum channel is represented by a maximally entangled Bell state. Teleportation has been demonstrated with the polarization photon [2] and a single coherent mode of field [3] in optical experiment. Recently, Gorbacher et. al [4] considered the quantum teleportation of twoparticle entangled state by three-particle entanglement, in their proposal, a three-particle Greenberger-Horne-Zeilinger [5] (GHZ) state is used as the communication channel. The three-photon GHZ state has been realized experimentlly [6]. In the proposal of standard teleportation, a Bell state or a GHZ state is used to as quantum channel for faithful teleportation. If the entangled state used as quantum channel is not maximally entangled, the faithful teleportation is not possible. Although Mor and Horodecki [7] showed that a " conclusive " teleportation of an unknown two-state particle can be made possible with pure entangled state of two-particle, whether a perfect teleportation of two-particle entangled state having a finite probability of success is possible with pure entangled state of three particles or not is unknown. In this report, a pure entangled three-particle state is considered, which is used as quantum channel. We present two schemes, by which, the probabilistic teleportations of two-particle entangled state can be realized. The successful probabilities of these two schemes are different. The probability of one scheme is equal to the twice modulus of the smaller coefficient of the pure entangled three-particle state.

Let a pure entangled three-particle state be in the following state

$$
|\Phi\rangle_{123}=\alpha|000\rangle_{123}+\beta|111\rangle_{123}
$$

where, $\alpha, \beta$ are reals, $|\alpha|>|\beta|$. The two-particle entangled state which will be teleported is:

$$
|\Psi\rangle_{45}=a|00\rangle_{45}+b|11\rangle_{45}
$$

The particle 3 of the state $|\Phi\rangle_{123}$ and particle pair $(4,5)$ belong to the sender Alice. The state of particle pair $(4,5)$ is unknown to Alice. Other two particles 1,2 belong to receiver 
Bob. In order to realize the teleportation, a Bell state measurement on particles 3 and 4 is made by Alice, which will project particles 1, 2 and 5 into the following state:

$$
\begin{aligned}
& \left\langle\left.\Phi^{ \pm}\right|_{34} \Phi\right\rangle_{123} \otimes|\Phi\rangle_{45}=\frac{\alpha a}{\sqrt{2}}|000\rangle_{125} \pm \frac{\beta b}{\sqrt{2}}|111\rangle_{125} \\
& \left\langle\left.\Psi^{ \pm}\right|_{34} \Phi\right\rangle_{123} \otimes|\Phi\rangle_{45}=\frac{\alpha b}{\sqrt{2}}|001\rangle_{125} \pm \frac{\beta a}{\sqrt{2}}|110\rangle_{125}
\end{aligned}
$$

where, $\left|\Phi^{ \pm}\right\rangle_{34}=\frac{1}{\sqrt{2}}\left[|00\rangle_{34} \pm|11\rangle_{34}\right],\left|\Psi^{ \pm}\right\rangle_{34}=\frac{1}{\sqrt{2}}\left[|01\rangle_{34} \pm|10\rangle_{34}\right]$. If Eq. (3) is obtained, then a unitary transformation on particle 5 is made in another basis $\{|x\rangle,|y\rangle\}$, where the new basis is related to the old basis $\{|0\rangle,|1\rangle\}$ in the following manner.

$$
\begin{aligned}
& |0\rangle=\alpha|x\rangle+\beta|y\rangle \\
& |1\rangle=\beta|x\rangle-\alpha|y\rangle
\end{aligned}
$$

which will transform Eq. (3) into the following:

$$
\frac{1}{\sqrt{2}}\left[\alpha^{2} a|00\rangle_{12} \pm \beta^{2} b|11\rangle_{12}\right]|x\rangle_{5}+\frac{\alpha \beta}{\sqrt{2}}\left[a|00\rangle_{12} \mp b|11\rangle_{12}\right]|y\rangle_{5}
$$

If Eq.(4) is obtained, by the same measurement on particle 5, it will be transformed into the following:

$$
\frac{-1}{\sqrt{2}}\left[\alpha^{2} b|00\rangle_{12} \mp \beta^{2} a|11\rangle_{12}\right]|y\rangle_{5}+\frac{\alpha \beta}{\sqrt{2}}\left[b|00\rangle_{12} \pm a|11\rangle_{12}\right]|x\rangle_{5}
$$

From the above analysis, we can see that if the result of measurement on particle 5 is $|x\rangle$ $(|y\rangle)$ in Eq. (7) (Eq. (8)), particles 1 and 2 are projected the state $\alpha^{2} a|00\rangle_{12} \pm \beta^{2} b|11\rangle_{12}($ $\alpha^{2} b|00\rangle_{12} \mp \beta^{2} a|11\rangle_{12}$ ), which can not be rotated back to the desired state without having any knowledge of the state parameters $a$ and $b$. The states created in Bob's hands depend not only on $\alpha$ and $\beta$, but also on $a$ and $b$. Unfortunately, $a$ and $b$ are supposed to be unknown, so it fails to reproduce the state exactly on Bob's side. That is means if the result of measurement on particle 5 is $|x\rangle(|y\rangle)$ in Eq. (7) (Eq. (8)), the teleportation fails. When the result of measurement on particle 5 is $|y\rangle(|x\rangle)$ in Eq.(7) (Eq.(8)), if a unitary operator 
performs on particles 1 and 2, then a perfect teleportation can be achieved. For example, if the result of Bell state measurement on particles 3 and 4 is $\left|\Phi^{+}\right\rangle_{34}$, the result of measurement on particle 5 is $|y\rangle$, then particles 1 and 2 are projected into state $a|00\rangle_{12}-b|11\rangle_{12}$. If we perform $U_{12}=\sigma_{1 z} \otimes I_{2}$ on particles 1 and 2, where $\sigma_{1 z}$ is Pauli operator performed on particle $1, I_{2}$ is a unity operator performed on particle 2 , then

$$
a|00\rangle_{12}-b|11\rangle_{12} \stackrel{U_{12}}{\rightarrow} a|00\rangle_{12}+b|11\rangle_{12}
$$

The right of Eq. (9) is desired state. From the above analysis, we can see that two measurements on Bob's side are needed. One is a Bell state measurement on particles 3 and 4 and the other is single particle Von Neumann measurement on particle 5 . The number of classical bits required is three. Two bits are required for Alice to inform Bob the result of a Bell measurement which will decide Bob's the unitary operation and the one more bit is required to tell Bob the result of single particle Von Neumann measurement which tells Bob whether a successful teleportation is possible. If a successful teleportation occurs, the unknown state can be reproduced on Bob's side with fidelity 1. The total probability of the successful teleportation is $2\left|\alpha^{2} \beta^{2}\right|$.

Next, we give another scheme, by which, the successful teleportation can be obtained with probability $2|\beta|^{2}$.

Similiarly, a Bell state measurement is performed by Alice on particles 3 and 4 of the state $|\Phi\rangle_{123} \otimes|\Phi\rangle_{45}$. When Eq. (3) is obtained, a unitary transformation is made on particle 5. To carry out this evolution, an auxiliary qubit with the original state $|0\rangle_{a}$ is introduced. Under the basis $\left\{|0\rangle_{1}|0\rangle_{a},|1\rangle_{1}|0\rangle_{a},|0\rangle_{1}|1\rangle_{a},|1\rangle_{1}|1\rangle_{a}\right\}$, a collective unitary transformation

$$
\left[\begin{array}{cccc}
\frac{\beta}{\alpha} & 0 & \sqrt{1-\frac{\beta^{2}}{\alpha^{2}}} & 0 \\
0 & 1 & 0 & 0 \\
0 & 0 & 0 & -1 \\
\sqrt{1-\frac{\beta^{2}}{\alpha^{2}}} & 0 & -\frac{\beta}{\alpha} & 0
\end{array}\right]
$$

is made, which will transform Eq.(3) to the result 


$$
\left[\frac{a \beta}{\sqrt{2}}|00\rangle_{12}|0\rangle_{5} \pm \frac{\beta b}{\sqrt{2}}|11\rangle_{12}|1\rangle_{5}\right]|0\rangle_{a}+\frac{\alpha a}{\sqrt{2}} \sqrt{1-\frac{\beta^{2}}{\alpha^{2}}}|00\rangle_{12}|0\rangle_{5}|1\rangle_{a}
$$

A measurement on auxiliary particle follows. The result $|1\rangle_{a}$ means the failed teleportation. If $|0\rangle_{a}$ is obtained, we make a measurement on particle 5 in another basis $\{|x\rangle,|y\rangle$, where is related to the old basis $\{|0\rangle,|1\rangle\}$ in the following manner

$$
\begin{aligned}
& |0\rangle=\frac{1}{\sqrt{2}}[|x\rangle+|y\rangle] \\
& |1\rangle=\frac{1}{\sqrt{2}}[|x\rangle-|y\rangle]
\end{aligned}
$$

This measurement will project the state of particles 1 and 2 into the following:

$$
\frac{\beta}{2}\left[a|00\rangle_{12} \pm b|11\rangle_{12}\right]|x\rangle_{5}+\frac{\beta}{2}\left[a|00\rangle_{12} \mp b|11\rangle_{12}\right]|y\rangle_{5}
$$

Obviously, whether the result $|x\rangle$ or $|y\rangle$ is obtained, if a unitary operation is made on particles 1 and 2, a perfect teleportation can be achieved. When Eq. (4) is obtained, it can be transformed to the result:

$$
\left[\frac{b \beta}{\sqrt{2}}|00\rangle_{12}|0\rangle_{5} \pm \frac{\beta a}{\sqrt{2}}|11\rangle_{12}|1\rangle_{5}\right]|0\rangle_{a}+\frac{\alpha b}{\sqrt{2}} \sqrt{1-\frac{\beta^{2}}{\alpha^{2}}}|00\rangle_{12}|1\rangle_{5}|1\rangle_{a}
$$

by the unitary transformation

$$
\left[\begin{array}{cccc}
0 & \frac{\beta}{\alpha} & \sqrt{1-\frac{\beta^{2}}{\alpha^{2}}} & 0 \\
1 & 0 & 0 & 0 \\
0 & 0 & 0 & -1 \\
0 & \sqrt{1-\frac{\beta^{2}}{\alpha^{2}}} & -\frac{\beta}{\alpha} & 0
\end{array}\right]
$$

Similarly, a measurement on auxiliary particle follows. Result $|1\rangle_{a}$ means failed teleportation .When result $|0\rangle_{a}$ is obtained, we also make a measurement on particle 5 in basis $\{|x\rangle,|y\rangle\}$, then Eq. (15) can be written as the following:

$$
\frac{\beta}{2}\left[b|00\rangle_{12} \pm a|11\rangle_{12}\right]|x\rangle_{5}+\frac{\beta}{2}\left[b|00\rangle_{12} \mp a|11\rangle_{12}\right]|y\rangle_{5}
$$


Similariy, if a unitary transformation is made on particles 1 and 2, a perfect teleportation can be achieved. In this scheme, Alice needs to do the follows for faithful teleportation. Firstly, she needs to make a Bell state measurement on particles 3 and 4, a unitary operation of two particles 5 and the auxiliary particle and a single qubit Von-Neumann measurement on auxiliary particle, then she will get some information by which she can judge whether the successful teleportation is possible or not. If successful teleportation is possible, she needs to make another single particle Von-Neumann measurement on particle 5. The number of classical bits is three if teleportation is failed or four if successful teleportation is possible. The total probability of successful teleportation is $2 \beta^{2}$, which is equal to twice modulus of the smaller coefficient of the pure entangled three-particle state. Contrast to the first scheme, this scheme is more complicited, it needs more quantum operations and more classical bits if the teleportation is successful. The advantage of this scheme is the probability of successful teleportation is higher than that of the first scheme.

In conclusion. In this report, two different probabilistic teleportation of a two-particle entangled state by pure entangled three-particle state are shown. The success probability of the first scheme is less than that of the second scheme. The successful probability of the second scheme is equal to the twice modulus of the smaller coefficient of the pure entangled three-particle state. The second scheme is more complicated than the first scheme.

This subject is supported by the National Natural Science Foundation of China and the Natioanl Natural Science Foundation for Youth of China. 


\section{REFERENCES}

[1] C. H. Bennett, et. al, Phys. Rev. Lett., 70, 1895 (1993)

[2] D. Bouwmeester, et. al., Nature, 390, 575 (1997)

[3] A. Furusawa, et. al, Science, 282, 706 (1998)

[4] V. N. Gorbachev and A. I. Trubilko, quant-ph 9906110

[5] M. Greenberger, M. A. Horne, A. Shimony and A. Zeilinger, Am. J. Phys, 58, 1131 (1990)

[6] D. Bouwmeester, et. al, Phys Rev. Lett. 82, 1345 (1999)

[7] T. Mor and P. Horodecki, quant-ph 9906039 